\title{
Context-free manifold calculus and the Fulton-MacPherson operad
}

\author{
VICTOR TURCHIN
}

The paper gives an explicit description of the Weiss embedding tower in terms of spaces of maps of truncated modules over the framed Fulton-MacPherson operad.

57Q45; 18D50, 55P48, 55P99

\section{Introduction}

It is well known that the $h$-principle fails for spaces of smooth maps avoiding singularities that depend on more than one point. The space $\operatorname{Emb}(M, N)$ of smooth embeddings between two manifolds is an example of such a space. However, the author believes that if one carefully constructs a space applying the $h$-principle taking into account configurations with less than or equal to $k$ points (in the source and target manifolds) then the obtained space will be exactly the $k^{\text {th }}$ approximation (to the space of maps avoiding the given multisingularities) arising from the Goodwillie-Weiss manifold calculus; see Weiss [25]. In this paper we demonstrate this idea for $\operatorname{Emb}(M, N)$. We produce an explicit model for $T_{k} \operatorname{Emb}(M, N)$, the $k^{\text {th }}$ stage of the Goodwillie-Weiss Taylor tower, as a space of $k$-tuples $\left(f_{i}: M^{\times i} \rightarrow N^{\times i}\right)_{i=1, \ldots, k}$ of equivariant (with respect to the symmetric group action) maps satisfying the natural conditions

- the preimage of the fat diagonal is the fat diagonal, and moreover the maps "behave nicely" when the points in configurations collide (approach the fat diagonal);

- the maps are compatible with respect to forgetting of points in configurations;

- in addition we also need to take into account the tangential data.

To address the first point above we replace $M^{\times i}$ and $N^{\times i}$ by the Axelrod-SingerFulton-MacPherson compactifications of configuration spaces of points in $M$ and $N$ respectively; see Axelrod and Singer [3] and Sinha [19]. The condition that the maps must be compatible with respect to forgetting of points is too restrictive-we make them compatible only up to some explicit homotopies that are made part of the data. Finally to take into account the tangential data, we add framing to the points in configurations. 
In other words maps $M^{\times i} \rightarrow N^{\times i}$ are replaced by sections of the space of $i$-multi 1 -jets. It is quite interesting that the solution to this geometric-homotopy problem that we produce actually has more of an algebraic flavor: the boundary conditions for the maps $f_{i}$ and for the homotopies are expressed as operad action conditions.

One should mention that another "geometrical model" of the Goodwillie-Weiss embedding tower was earlier produced by Goodwillie, Klein, and Weiss in [13]. In their model the maps $f_{i}: M^{\times i} \rightarrow N^{\times i}$ must be smooth (unlike our model where only continuity is required). These maps must satisfy some natural equivariance and transversality conditions with respect to the fat diagonal. The tangential data in that model are encoded by the behavior of $f_{2}$ near the diagonal. The compatibility with respect to forgetting of points in configurations is treated similarly: compatibility being replaced by compatibility up to homotopy. Even though their model seems similar to the one produced in this paper, they are still quite different in spirit. In particular the author can not see any direct map or a natural short zigzag between the two models.

\section{Organization of the paper}

In Section 1 we outline a general framework of context-free manifold calculus and its connection to the framed discs operad. The details of this approach were completed by $\mathrm{P}$ Boavida de Brito and $\mathrm{M}$ Weiss in [5]. One of the main results of their work is Theorem 1.5 that describes the Weiss-Taylor tower of a context-free topological presheaf on a manifold in terms of maps of truncated right modules over the framed discs operad. Section 1 is given to emphasize the fact that for this description one can use the operad of framed discs with both its usual and discrete topology. A discrete version of Theorem 1.5 appeared earlier in a work of G Arone and the author [2]. Section 2 is where the main construction is given. In Theorem 2.1 we replace the framed discs operad by the framed Fulton-MacPherson operad and describe Weiss' approximations $T_{k} \operatorname{Emb}(M, N)$ to the space $\operatorname{Emb}(M, N)$ of embeddings of one manifold into another in terms of maps of truncated right modules over the latter operad. The right modules in question themselves are naturally obtained from the Axelrod-SingerFulton-MacPherson compactifications of framed configuration spaces in manifolds $M$ and $N$. This description of the embedding tower resembles both the GoodwillieKlein-Weiss construction [13] mentioned above and also Sinha's models [21; 20] for spaces of one dimensional knots. The proof of Theorem 2.1 is very straightforward and does not rely on the Boavida-Weiss Theorem 1.5. Sections 3 and 4 do this job. Moreover our construction can be used to give an alternative proof of Theorem 1.5 which is shown in Section 5. Section 6 produces another application of our construction describing Weiss' tower for spaces of long embeddings in terms of maps of truncated infinitesimal bimodules over the Fulton-MacPherson operad. As a corollary we obtain 
that for $n>m+2$ the space $\overline{\operatorname{Emb}}_{c}\left(\mathbb{R}^{m}, \mathbb{R}^{n}\right)$ is equivalent to the space of derived maps between the operads of little discs $\mathcal{B}_{m}$ and $\mathcal{B}_{n}$ in the category of infinitesimal bimodules over $\mathcal{B}_{m}$ which can be seen as a high-dimensional generalization of Sinha's work [20].

As a general remark the category Top of topological spaces that we consider is the category of compactly generated Hausdorff spaces with the usual model structure with weak equivalences and Serre fibrations. We also consider the model structure on the categories of (truncated) right modules and (truncated) infinitesimal bimodules over different operads. The idea is that each such structure is equivalent to a functor from a certain category enriched in Top. The categories encoding such structures were explicitly described by Arone and the author in [2]. We use the projective model structure on the categories of such functors with equivalences and fibrations being objectwise equivalences and objectwise fibrations.

\section{Context-free manifold calculus and the operad of framed discs}

In [25] M Weiss introduced the so called manifold calculus of functors. Given a smooth manifold $M$, denote by $\mathcal{O}(M)$ the category of open subsets of $M$. For any isotopy invariant cofunctor $F: \mathcal{O}(M) \rightarrow$ Top in topological spaces, Weiss defines a Taylor tower

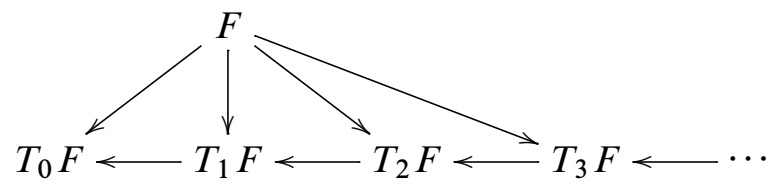

of polynomial approximations of $F$. It became clear a while ago that the manifold calculus of functors is deeply related to the operad of little discs. Below we outline one of the constructions that shows this connection.

Let $\operatorname{Man}_{m}$ denote the category of smooth $m$-manifolds, where the morphisms are codimension zero embeddings. This category is naturally enriched in topological spaces. We denote by ${ }^{\delta} \operatorname{Man}_{m}$ its discretization. For any $m$-manifold $M$ one has an obvious forgetful functor

$$
I_{M}: \mathcal{O}(M) \rightarrow{ }^{\delta} \mathbb{M a n}_{m}
$$

Definition 1.1 A cofunctor $\widetilde{F}: \mathcal{O}(M) \rightarrow$ Top is context-free if $\widetilde{F}$ up to a natural equivalence factors through ${ }^{\delta} \mathbb{M a n}_{m}$. In other words $\tilde{F} \simeq F \circ I_{M}$ for some cofunctor $F:{ }^{\delta} \operatorname{Man}_{m} \rightarrow$ Top. 
In the sequel by a context-free cofunctor we will often understand the underlying cofunctor $F:{ }^{\delta} \operatorname{Man}_{m} \rightarrow$ Top. Notice that this definition is slightly different and somewhat simpler than the one previously used; see [2, Definition 4.9]. But the idea is still the same-a cofunctor is context-free if "it does not depend" on where the open subsets are located. As an example, consider a nontrivial fibration $p: E \rightarrow M$; then the cofunctor $\Gamma(-, p)$ of continuous sections of $p$ is linear, but in general not context-free. The context-free cofunctors abound. The embedding and immersion cofunctors $\operatorname{Emb}(-, N), \operatorname{Imm}(-, N)$ are context-free. These cofunctors assign to an open set $U \subset M$ the space of smooth embeddings, respectively immersions, of $U$ in another smooth manifold $N$ of dimension greater than or equal to $m$. As a further generalization for any type of multisingularity $\mathbb{S}$ the spaces $\operatorname{Maps}_{\mathbb{S}}(M, N)$ of smooth maps $M \rightarrow N$ that avoid $\mathbb{S}$ also define a context-free cofunctor

$$
\operatorname{Maps}_{\mathbb{S}}(-, N): \mathbb{M a n}_{m} \rightarrow \text { Top. }
$$

All these cofunctors are in fact continuous in the sense that they are defined on the enriched category $\operatorname{Man}_{m}$. Given a context-free cofunctor it is natural to forget about the initial manifold $M$ and study the calculus of cofunctors with domain ${ }^{\delta} \operatorname{Man}_{m}$. We will call such calculus context-free manifold calculus. This variation of manifold calculus is actually more similar to their brothers homotopy calculus (see Goodwillie $[10 ; 11 ; 12]$ ) and orthogonal calculus (see Weiss [24]) since it deals with all manifolds similarly as the homotopy calculus deals with all topological spaces or spectra, and the orthogonal calculus deals with all vector spaces of finite dimension.

By an obvious analogy with [25] define a Grothendieck topology $\mathcal{J}_{k}$ on ${ }^{\delta} \mathbb{M a n}_{m}$ in which $\left\{f_{i}: U_{i} \hookrightarrow V\right\}_{i \in I}$ is a $\mathcal{J}_{k}$-cover if and only if $\bigcup_{i \in I} f_{i}\left(U_{i}\right)^{\times k}=V^{\times k}$. In other words any configuration of less than or equal to $k$ points in $V$ should appear in the image of at least one $U_{i}$. For our purposes we will be using the following definition of polynomial functors. It is a nontrivial result of Weiss that the following is equivalent to a more usual definition due to Goodwillie that uses cubical diagrams [25; 26].

Definition 1.2 An isotopy invariant presheaf $F:{ }^{\delta} \mathbb{M a n}_{n} \rightarrow$ Top is called polynomial of degree less than or equal to $k$ if it is a homotopy $\mathcal{J}_{k}$-sheaf.

A reader unfamiliar with the notion of a homotopy sheaf may wait until Section 3 where we explain what this property means.

Let ${ }^{\delta} \mathcal{O}_{\leq k}$ (respectively $\mathcal{O}_{\leq k}$ ) be the full subcategory of ${ }^{\delta} \operatorname{Man}_{m}$ (respectively $\operatorname{Man}_{m}$ ) whose objects are disjoint unions of less than or equal to $k$ standard $m$-balls. Thus this category has only $k+1$ objects. Define $T_{k} F$ as the homotopy right Kan extension 
of $F$ from ${ }^{\delta} \mathcal{O}_{\leq k}$ to ${ }^{\delta} \operatorname{Man}_{m}$ :

$$
T_{k} F(M)=\operatorname{holim}_{\delta \mathcal{O}_{\leq k} \downarrow M} F .
$$

For every $M$ one has a natural map $F(M) \rightarrow T_{k} F(M)$. Denote by $\eta_{F}$ the corresponding natural transformation.

Proposition 1.3 For any isotopy invariant presheaf $F:{ }^{\delta} \mathbb{M a n}_{m} \rightarrow$ Top the natural transformation $\eta_{F}: F \rightarrow T_{k} F$ is a homotopy $\mathcal{J}_{k}$ sheafification of $F$ in the sense that

- $T_{k} F$ is polynomial of degree less than or equal to $k$;

- in case $F$ is polynomial of degree less than or equal to $k$ then $\eta_{F}$ is a natural equivalence.

Proof One obviously has that $F$ is polynomial of degree less than or equal to $k$ if and only if its restriction on $\mathcal{O}(M)$ is so for all $M \in{ }^{\delta} \mathbb{M a n}_{m}$. Let $\mathcal{O}_{\leq k}(M)$ denote the subcategory of open subsets of $M$ diffeomorphic to a disjoint union of less than or equal to $k$ balls. One has a natural evaluation functor

$$
\operatorname{ev}_{M}:{ }^{\delta} \mathcal{O}_{\leq k} \downarrow M \rightarrow \mathcal{O}_{\leq k}(M)
$$

that assigns to any embedding $f: U \hookrightarrow M, U \in{ }^{\delta} \mathcal{O}_{\leq k}$, its image $f(U)$. This cofunctor is homotopy right cofinal ${ }^{1}$ and therefore the induced map

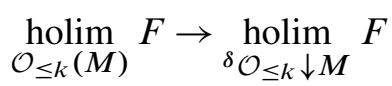

is a weak equivalence. Notice that the first homotopy limit is exactly Weiss' formula for the $k^{\text {th }}$ approximation. Thus the properties of $T_{k} F$ mentioned in the proposition follow from the analogous properties of Weiss' approximations [25]. The cofinality of $\mathrm{ev}_{M}$ is immediate from the fact that for any $V \in \mathcal{O}_{\leq k}(M)$ the corresponding undercategory $V \downarrow \mathrm{ev}_{M}$ has initial objects.

There is yet another way to describe $T_{k} F$.

Lemma 1.4 For any presheaf $F:{ }^{\delta} \operatorname{Man}_{m} \rightarrow$ Top one has a natural equivalence

$$
\underset{\delta_{\mathcal{O}_{\leq k} \downarrow M}^{\operatorname{holim}}}{ } F \simeq \operatorname{hNat}_{\delta_{\mathcal{O}}}\left({ }^{\delta} \operatorname{Emb}(\cdot, M), F(\cdot)\right) .
$$

\footnotetext{
${ }^{1}$ Actually it is both right and left cofinal, but we care only about the right cofinality since we only need that $\mathrm{ev}_{M}$ preserves homotopy limits and our functors are contravariant.
} 
In the above ${ }^{\delta} \operatorname{Emb}(\cdot, M)$ is a cofunctor that assigns to any $U \in{ }^{\delta} \mathcal{O}_{\leq k}$ the space of embeddings $\operatorname{Emb}(U, M)$ with the discrete topology; hNat denotes the space of homotopy natural transformations between cofunctors on ${ }^{\delta} \mathcal{O}_{\leq k}$. This lemma is a particular case of Arone [1, Lemma 3.7]. The idea of the proof is that both spaces can be described as a totalization of certain cosimplicial spaces, and moreover the second corresponding cosimplicial space is obtained by an edgewise subdivision of the first one. Thus the equivalence (1-3) can be viewed as a natural homeomorphism. The latter description of $T_{k} F$ has a nice interpretation from the point of view of the theory of operads. Notice that the category $\operatorname{Man}_{m}$, respectively ${ }^{\delta} \mathbb{M a n}_{m}$, is symmetric monoidal where the monoidal structure is given by disjoint union, and unit is the empty set. Let $\mathcal{E}\left(D^{m}\right)$, respectively ${ }^{\delta} \mathcal{E}\left(D^{m}\right)$, denote the operad of endomorphisms of the unit disc $D^{m}$ in $\operatorname{Man}_{m}$, respectively ${ }^{\delta} \mathbb{M a n}_{m}$. It is obvious that $\mathcal{E}\left(D^{m}\right)$ is equivalent to the operad of framed discs, and ${ }^{\delta} \mathcal{E}\left(D^{m}\right)$ is simply the discretization of $\mathcal{E}\left(D^{m}\right)$. Next notice that a cofunctor $G: \mathcal{O}_{\leq k} \rightarrow$ Top, respectively $G:{ }^{\delta} \mathcal{O}_{\leq k} \rightarrow$ Top, is exactly the same thing as a $k$-truncated right module over $\mathcal{E}\left(D^{m}\right)$, respectively ${ }^{\delta} \mathcal{E}\left(D^{m}\right)$. Indeed, given such functor define a sequence of $k+1$ spaces $G(i):=G\left(\coprod_{i} D^{m}\right)$, $i=0, \ldots, k$. This sequence has an obvious $k$-truncated right action of $\mathcal{E}\left(D^{m}\right)$, respectively ${ }^{\delta} \mathcal{E}\left(D^{m}\right)$. This is a general fact since the operad in question is the operad of endomorphisms of $D^{m}$ and $G(\cdot)$ is a sequence of values of a cofunctor on the monoidal powers of $D^{m}$. Thus $T_{k} F(M)$ can be described as the space of derived maps of $k$-truncated right modules over ${ }^{\delta} \mathcal{E}\left(D^{m}\right)$ :

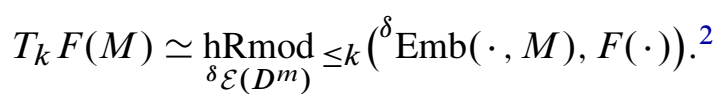

It is natural to ask whether ${ }^{\delta} \mathcal{E}\left(D^{m}\right)$ can be replaced by $\mathcal{E}\left(D^{m}\right)$ in case $F$ is continuous. I asked this question to $\mathrm{M}$ Weiss and it turned out that his student $\mathrm{P}$ Boavida de Brito was already working on the same problem and a few months later they found an elegant solution thus proving the following.

Theorem 1.5 [5] For a cofunctor $F: \mathbb{M a n}_{m} \rightarrow$ Top the natural composition

$(1-5) \quad F(M) \longrightarrow \mathrm{Rmod}_{\mathcal{E}\left(D^{m}\right)}(\operatorname{Emb}(\cdot, M), F(\cdot)) \longrightarrow \operatorname{hRmod}_{\mathcal{E}\left(D^{m}\right)}(\operatorname{Emb}(\cdot, M), F(\cdot))$ is equivalent to the homotopy $\mathcal{J}_{k}$-sheafification $F(M) \rightarrow T_{k} F(M)$.

They used a slightly different language to formulate this result, but their [5, Section 6] shows that it can be reformulated using the operadic approach. A discrete version of

\footnotetext{
${ }^{2}$ As a general remark regarding notation, for a right module $G(\cdot)$ we will denote by $G\left(\left.\cdot\right|_{\leq k}\right)$ its $k-$ truncation. However if we consider the space of (derived) maps of truncated right modules the notation $\left.\right|_{\leq k}$ will be dropped since $\mathrm{Rmod}_{\leq k}$ already indicates that the objects are truncated.
} 
this result appeared in [2] with the only difference that in that paper we considered only submanifolds of $\mathbb{R}^{m}$ and the acting operad was the operad of little (nonframed) discs. The above result is quite remarkable not only because it gives a connection between the theory of operads and manifold calculus, but also it shows that the discretization of the operad of (framed) discs still keeps a lot of information about the initial topological operad. It would be interesting to understand exactly what information is preserved by discretization and to which extent it is true for any or a larger range of topological operads. As a natural analogy the homology of any Lie group with coefficients in a cyclic group is conjectured to coincide with the homology of its discretization. This conjecture, known as the Friedlander-Milnor conjecture, was the subject of extensive research; see Friedlander and Mislin [8], Milnor [15], Morel [16], Sah [17] and Suslin [22].

\section{Embedding tower and Fulton-MacPherson operad}

The motivating example for the Weiss manifold calculus is the study of embedding spaces. The Taylor tower (1-1) for the embedding cofunctor $\operatorname{Emb}(-, N)$ is usually called embedding tower. It turns out that one can obtain a nice description of the embedding tower by replacing the operad $\mathcal{E}\left(D^{m}\right)$ by a much smaller but equivalent operad $\mathcal{F}_{m}^{f r}$ the so called framed Fulton-MacPherson operad. Recall the FultonMacPherson operad $\mathcal{F}_{m}$ (see Getzler and Jones [9] and Salvatore [18]). This operad was simultaneously introduced by several people, in particular by Getzler and Jones [9]. Its components are manifolds with corners such that the interior of $\mathcal{F}_{m}(k)$ is $C\left(k, \mathbb{R}^{m}\right) / G$ the configuration space of $m$ distinct labeled points in $\mathbb{R}^{m}$ quotiented out by translations and positive rescalings. Notice that $\mathcal{F}_{m}(0)=\mathcal{F}_{m}(1)=\{*\}$. By reduced Fulton-MacPherson operad $\overline{\mathcal{F}}_{m}$ we will understand the suboperad of $\mathcal{F}_{m}$ obtained by making the degree zero component to be empty $\overline{\mathcal{F}}_{m}(0)=\varnothing$ and keeping all the other components the same $\overline{\mathcal{F}}_{m}(k)=\mathcal{F}_{m}(k), k \geq 1$. It is noticed in [9] that the operad $\overline{\mathcal{F}}_{m}$ is cofibrant. As an operad in sets it is freely generated by the interiors of its components. The framed Fulton-MacPherson operad $\mathcal{F}_{m}^{f r}$ has components $\mathcal{F}_{m}(k) \times\left(\mathrm{GL}_{m}\right)^{\times k}, k \geq 0$, where $\mathrm{GL}_{m}$ is the group of general linear transformations of $\mathbb{R}^{m}$. Each component of this operad is a certain compactification of the space of framed configurations modulo translations and rescaling. The composition in $\mathcal{F}_{m}^{f r}$ uses the fact that $\mathcal{F}_{m}$ is an operad in spaces with $\mathrm{GL}_{m}$-action; see [18]. We will also consider the oriented framed Fulton-MacPherson operad $\mathcal{F}_{m}^{o r}$ which is a suboperad of $\mathcal{F}_{m}^{f r}$ and whose components are $\mathcal{F}_{m}(k) \times\left(\mathrm{GL}_{m}^{+}\right)^{\times k}, k \geq 0$, where $\mathrm{GL}_{m}^{+}$is the group of orientation preserving linear transformations of $\mathbb{R}^{m}$.

For any manifold $M$ let $C(k, M), k \geq 0$, denote the configuration space

$$
C(k, M)=\left\{\left(x_{1}, \ldots, x_{k}\right) \in M^{\times k} \mid x_{i} \neq x_{j} \text { for all } i \neq j\right\} .
$$


Let $C[k, M]$ denote its Axelrod-Singer-Fulton-MacPherson compactification [3; 19]. A thorough treatment of this construction is given by Sinha in [19] from where we borrowed our notation. $C[k, M]$ is a manifold with corners whose interior is $C(k, N)$. The boundary strata consist of configurations where some of the points collided. One has an obvious projection $C[k, M] \rightarrow M^{\times k}$ and we denote by $p_{i}: C[k, M] \rightarrow M$ its $i^{\text {th }}$ component, $1 \leq i \leq n$. In case a manifold $N$ has dimension greater than or equal to $m$ we define a space $C^{m \_}{ }^{m}[k, N]$ which fibers over $C[k, N]$ with a fiber over any point $X \in C[k, N]$ being the space of tuples $\left(\alpha_{1}, \ldots, \alpha_{k}\right)$, where each $\alpha_{i}: \mathbb{R}^{m} \hookrightarrow T_{p_{i}(X)} M$ is a linear injective map called partial framing. In case of a manifold of dimension $m$ we will simply write $C^{f r}[k, M]$ instead of $C^{m} f r[k, M]$. In case $M$ is oriented we also consider spaces $C^{\text {or }}[k, M] \subset C^{f r}[k, M]$ for which all the framings $\alpha_{i}: \mathbb{R}^{m} \rightarrow T_{p_{i}(X)} M$ are orientation preserving. The sequences $C^{f r}[\cdot, M]$, $C^{m_{-} f r}[\cdot, N]$ are right modules over $\mathcal{F}_{m}^{f r}$. The degree zero component $\mathcal{F}_{m}^{f r}(0)$ acts by forgetting points in configurations. Any other element $x \in \mathcal{F}_{m}^{f r}(k), k \geq 1$, acts by replacing a point in a configuration by the infinitesimal configuration $x$ according to the framing. Similarly, for an oriented $m$-manifold $M$, the sequence $C^{\text {or }}[\cdot, M]$ is naturally a right module over $\mathcal{F}_{m}^{o r}$; and for a parallelized $M, C[\cdot, M]$ is a right module over $\mathcal{F}_{m}$. Notice that any embedding $f: M \hookrightarrow N$ induces a natural evaluation map ev $f: C^{f r}[k, M] \rightarrow C^{m \_f r}[k, N]$ which is a morphism of right $\mathcal{F}_{m}^{f r}$-modules.

Theorem 2.1 In the above notation the composition

$$
\begin{aligned}
\operatorname{Emb}(M, N) & \stackrel{\mathrm{ev}}{\rightarrow} \operatorname{Rmod}_{\mathcal{F}_{m}^{f r}}\left(C^{f r}[\cdot, M], C^{m_{-} f r}[\cdot, N]\right) \\
& \rightarrow \underset{\mathcal{F}_{m}^{f r}}{\mathrm{hRmod}_{\leq k}}\left(C^{f r}[\cdot, M], C^{m_{-} f r}[\cdot, N]\right)
\end{aligned}
$$

is equivalent to the $\mathcal{J}_{k}$-sheafification $\operatorname{Emb}(M, N) \rightarrow T_{k} \operatorname{Emb}(M, N)$. In particular the limit of the embedding tower $T_{\infty} \operatorname{Emb}(M, N)$ is equivalent to the space of derived maps of right $\mathcal{F}_{m}^{f r}$-modules

$$
T_{\infty} \operatorname{Emb}(M, N) \simeq \underset{\mathcal{F}_{m}^{f r}}{\operatorname{hRmod}}\left(C^{f r}[\cdot, M], C^{m_{-} f r}[\cdot, N]\right) .
$$

In case $M$ is oriented, respectively parallelized, the same is true for the compositions

$$
\begin{gathered}
\operatorname{Emb}(M, N) \longrightarrow \operatorname{h}_{\mathcal{F}_{m}^{o r}} \bmod _{\leq k}\left(C^{o r}[\cdot, M], C^{m_{-} f r}[\cdot, N]\right), \\
\operatorname{Emb}(M, N) \longrightarrow \operatorname{hRmod}_{\mathcal{F}_{m}}\left(C[\cdot, M], C^{m_{-} f r}[\cdot, N]\right) .
\end{gathered}
$$

Remark 2.2 The first statement of this theorem is equivalent to the Boavida-Weiss Theorem 1.5 applied to special case of the embedding cofunctor; see Section 5.2. 
Moreover our construction can be used to give an alternative proof of Theorem 1.5 itself; see Section 5.1.

To prove Theorem 2.1 we will construct a cofibrant replacement $\widetilde{C}^{f r}[\cdot, M]$ (functorial in $M$ ) of $C^{f r}[\cdot, M]$ in the category of right modules over $\mathcal{F}_{m}^{f r}$. The following result is important to understand that construction.

Lemma 2.3 For any smooth $m$-manifold $M, C^{f r}[\cdot, M]$ is cofibrant in the category of right modules over the reduced framed Fulton-MacPherson operad $\overline{\mathcal{F}}_{m}^{f r}$. Similarly any $k$-truncation $C^{f r}\left[\left.\cdot\right|_{\leq k}, M\right]$ is cofibrant in the category of $k$-truncated right modules.

Proof Intuitively one can see that $C^{f r}[\cdot, M]$ is cofibrant since as a right $\overline{\mathcal{F}}_{m}^{f r}$ module in sets it is freely generated by the symmetric sequence $C(\cdot, M)$-the interiors of $C[\cdot, M]$. In the sequel each $C(k, M), k \geq 0$, will be called a generating stratum of this right module. Below we give a more rigorous argument.

Let $\operatorname{Sub}_{\leq k}(M)$ denote the space of subsets of $M$ of cardinality less than or equal to $k$, topologized as a quotient of $\{\varnothing\} \amalg M^{\times k} / \Sigma_{k}$. We will also consider the space of all finite subsets of $M$,

$$
\operatorname{Sub}(M)=\bigcup_{k \geq 0} \operatorname{Sub}_{\leq k}(M)
$$

Denote by $g$ the composition

$$
g: \coprod_{k=0}^{\infty} C^{f r}[k, M] \longrightarrow \coprod_{k=0}^{\infty} M^{\times k} \longrightarrow \operatorname{Sub}(M) .
$$

For $X \in C^{f r}[k, M]$ we say that $g(X)$ is the set of geometrically distinct points of $X$. Define a filtration in $C^{f r}[\cdot, M]$ by the number of geometrically distinct points $C_{(\ell)}^{f r}[\cdot, M]=g^{-1}\left(\operatorname{Sub}_{\leq \ell}(M)\right)$ :

$$
C_{(0)}^{f r}[\cdot, M] \subset C_{(1)}^{f r}[\cdot, M] \subset C_{(2)}^{f r}[\cdot, M] \subset \cdots .
$$

Notice that the right action of $\overline{\mathcal{F}}_{m}^{f r}$ does not change the image of $g$ :

$$
g\left(X \circ_{i} c\right)=g(X)
$$

for all $X \in C^{f r}[\cdot, M]$ and $c \in \overline{\mathcal{F}}_{m}^{f r}(\cdot)$. Therefore filtration (2-4) is a filtration of right modules over $\overline{\mathcal{F}}_{m}^{f r}$. One can show that each inclusion in this filtration is a cofibration, which guarantees that all elements of the filtration and its colimit are cofibrant. In 
case $M$ is parallelized one has the following pushout square of right $\overline{\mathcal{F}}_{m}^{f r}$-modules:

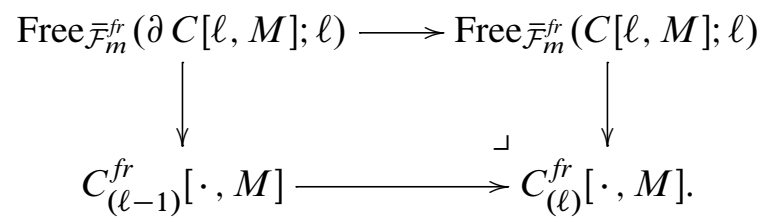

In the above Free $\overline{\mathcal{F}}_{m}^{f r}(A ; \ell)$ denotes the free $\overline{\mathcal{F}}_{m}^{f r}$ right module generated by a space $A$ with a free $\Sigma_{\ell}$ action; $\partial C[\ell, M]$ denotes the boundary of $C[\ell, M]$. The vertical maps above are defined by a choice of trivialization of $T M$. Since the upper arrow is a cofibration so is the lower one.

If $M$ is not parallelized, consider a cellular decomposition of $M$ and then refine the above argument using the trivialization of the tangent bundle over each cell.

The above lemma shows that $C^{f r}[\cdot, M]$ fails to be cofibrant as a right $\mathcal{F}_{m}^{f r}$ module only because of the degree zero component $\mathcal{F}_{m}^{f r}(0)=*$ which acts by forgetting the corresponding point in configuration. A slight adjustment has to be done in order to make it cofibrant.

We define $\tilde{C}^{f r}[k, M]$ as a space of hairy configurations. Its points are the tuples $\left(X ; y_{1}, \ldots, y_{\ell} ; t_{1}, \ldots t_{\ell}\right), \ell \geq 0$, where $X \in C^{f r}[k, M], y_{i} \in M, i=1, \ldots, \ell$ and $0 \leq t_{1} \leq t_{2} \leq \cdots \leq t_{\ell} \leq 1$. The data $(\bar{y} ; \bar{t})=\left(y_{1}, \ldots, y_{\ell} ; t_{1}, \ldots, t_{\ell}\right)$ can be viewed as a bunch of hairs that grow from the points $y_{1}, \ldots, y_{\ell}$ and have length $t_{1}, \ldots, t_{\ell}$ respectively. Thus

$$
\widetilde{C}^{f r}[k, M]=\left(\coprod_{\ell=0}^{\infty} C^{f r}[k, M] \times\left(M^{\times \ell} \times[0,1]^{\ell}\right) / \Sigma_{\ell}\right) / \sim,
$$

where the equivalence relation is as follows: If one of the hairs (say $y_{1}$ ) gets contracted to zero, the corresponding point $y_{1}$ disappears. If two hairy points $y_{i}$ and $y_{j}$ collide, only the hair of the longer length survives. If one of the hairs collides with a point or a conglomeration of points of $X$, the hair also disappears. Explicitly,

$$
\left(X ; y_{1}, \ldots, y_{\ell} ; t_{1}, \ldots, t_{\ell}\right) \sim\left(X ; y_{\sigma_{1}}, \ldots, y_{\sigma_{\ell}} ; t_{\sigma_{1}}, \ldots, t_{\sigma_{\ell}}\right),
$$

whenever $t_{\sigma_{1}} \leq \cdots \leq t_{\sigma_{\ell}}, \sigma \in \Sigma_{\ell}$;

$$
\begin{aligned}
& \left(X ; y_{1}, y_{2}, \ldots, y_{\ell} ; 0, t_{2}, \ldots, t_{\ell}\right) \sim\left(X ; y_{2}, \ldots, y_{\ell} ; t_{2}, \ldots, t_{\ell}\right), \\
& \left(X ; y_{1}, \ldots, y_{\ell} ; t_{1}, \ldots, t_{\ell}\right) \sim\left(X ; y_{1}, \ldots, \widehat{y}_{i}, \ldots, y_{\ell} ; t_{1}, \ldots, \widehat{t}_{i}, \ldots, t_{\ell}\right),
\end{aligned}
$$

whenever $y_{i}=y_{j}, i<j$, or $y_{i}=p_{m}(X), 1 \leq m \leq k$. 
The right action of any element $c \in \mathcal{F}_{m}^{f r}(k)$ in degree $k \geq 1$, is defined to affect only $X$ :

$$
(x ; \bar{y} ; \bar{t}) \circ_{i} c=\left(x \circ_{i} c ; \bar{y} ; \bar{t}\right) .
$$

For $\{e\}=\mathcal{F}_{m}^{f r}(0)$ the right action is defined by

$$
(X ; \bar{y} ; \bar{t}) \circ_{i} e=\left(X \circ_{i} e ; \bar{y}, p_{i}(X) ; \bar{t}, 1\right) .
$$

In other words this action replaces the $i^{\text {th }}$ point in $X$ by a hair of length one. Notice however that in the case that $p_{i}(X)=p_{j}(X)$ for some $j \neq i$, one has that $\left(X \circ_{i}\right.$ $\left.e ; \bar{y}, p_{i}(X) ; \bar{t}, 1\right)=\left(X \circ_{i} e ; \bar{y} ; \bar{t}\right)$ by $(2-10)$.

Proposition 2.4 The natural projection

$$
\widetilde{C}^{f r}[\cdot, M] \rightarrow C^{f r}[\cdot, M],
$$

that forgets all hairs, defines a cofibrant replacement of $C^{f r}[\cdot, M]$ as a right $\mathcal{F}_{m}^{f r}$ module. Moreover for every $k \geq 0$ the $k^{\text {th }}$ truncation $\widetilde{C}_{(k)}^{f r}\left[\left.\cdot\right|_{\leq k}, M\right]$ of the $k^{\text {th }}$ filtration term (2-16) is a cofibrant replacement of $C^{f r}\left[\left.\cdot\right|_{\leq k}, M\right]$ as a $k$-truncated right $\mathcal{F}_{m}^{f r}$ module.

By an obvious analogy we define $\widetilde{C}^{o r}[\cdot, M]$, respectively $\widetilde{C}[\cdot, M]$, as a cofibrant replacement of $C^{\text {or }}[\cdot, M]$, respectively $C[\cdot, M]$, in the category of right modules over $\mathcal{F}_{m}^{o r}$, respectively $\mathcal{F}_{m}$.

Sketch of the proof First we notice that the projection (2-13) is an equivalence of $\mathcal{F}_{m}^{f r}$-modules. This means that for every component $\cdot=\ell$ this map is a $\Sigma_{\ell-}$ equivariant homotopy equivalence. The homotopy inverse is the natural inclusion $C^{f r}[\ell, M] \hookrightarrow \widetilde{C}^{f r}[\ell, M]$

Second, it is easy to see that as a right $\mathcal{F}_{m}$-module in sets $\widetilde{C}^{f r}[\cdot, M]$ is freely generated by the symmetric sequence

$$
\widetilde{C}(\ell, M)=\coprod_{i=0}^{+\infty} C(\ell+i, M) \times(0,1)^{i} / \Sigma_{i}, \quad \ell \geq 0,
$$

where $\Sigma_{i}$ acts by a simultaneous permutation of the last $i$ points in $C(\ell+i, M)$ and the coordinates of $(0,1)^{i}$. We sketch a proof below that shows in which order the above generating strata are attached. For simplicity one can assume that $M$ is parallelized. If 
it is not the argument must be further refined as in the proof of Lemma 2.3. Define a map

$$
\tilde{g}: \coprod_{\ell=0}^{+\infty} \widetilde{C}^{f r}[\ell, M] \rightarrow \operatorname{Sub}(M)
$$

by sending $\tilde{X}=\left(X ; y_{1} \cdots y_{i} ; t_{1} \cdots t_{i}\right)$, with all $t_{j} \neq 0$, to $g(X) \bigcup\left\{y_{1} \cdots y_{i}\right\}$. Notice that $\tilde{g}$ is not continuous contrary to the map $g$. Again $\widetilde{g}(\tilde{X})$ is called the set of geometrically distinct points of $\tilde{X}$. Notice that this map is invariant with respect to the $\mathcal{F}_{m}^{f r}$ action:

$$
\widetilde{g}\left(\tilde{X} \circ_{i} c\right)=\widetilde{g}(\tilde{X})
$$

for every $X \in \widetilde{C}^{f r}[\cdot, M]$ and $c \in \mathcal{F}_{m}^{f r}$. In particular this means we can define a filtration of right $\mathcal{F}_{m}^{f r}$-modules:

$$
\widetilde{C}_{(0)}^{f r}[\cdot, M] \subset \widetilde{C}_{(1)}^{f r}[\cdot, M] \subset \widetilde{C}_{(2)}^{f r}[\cdot, M] \subset \cdots,
$$

where $\widetilde{C}_{(k)}^{f r}[\cdot, M]$ is the preimage $\tilde{g}^{-1}\left(\operatorname{Sub}_{\leq k}(M)\right)$. Each inclusion in the above filtration is a cofibration of right $\mathcal{F}_{m}^{f r}$ modules. To see this we notice that besides the filtration by the number of geometrically distinct points there is another natural filtration in $\widetilde{C}^{f r}[\cdot, M]$ by the number of hairs whose length is strictly between 0 and 1 . Let $\widetilde{C}_{(k), i}^{f r}[\cdot, M], k \geq 0, i \geq-1$, denote the right submodule of $\widetilde{C}_{(k+1)}^{f r}[\cdot, M]$ that consists of hairy configurations with either less than or equal to $k$ geometrically distinct points or with exactly $k+1$ geometrically distinct points, but with less than or equal to $i$ hairs of length strictly between 0 and 1 . Notice that

$$
\widetilde{C}_{(k), k+1}^{f r}[\cdot, M]=\widetilde{C}_{(k+1)}^{f r}[\cdot, M]=\widetilde{C}_{(k+1),-1}^{f r}[\cdot, M] .
$$

Thus the filtration by the geometrically distinct points and by the number of hairs of length less than 1 can be considered as a refinement of (2-16). One can easily see that the generating stratum $C(\ell+i, M) \times(0,1)^{i} / \Sigma_{\ell}$ from (2-14) is attached exactly when we pass from $\widetilde{C}_{(\ell+i-1), i-1}^{f r}[\cdot, M]$ to $\widetilde{C}_{(\ell+i-1), i}^{f r}[\cdot, M]$. By this we mean that one has a pushout square similar to (2-6):

$$
\begin{gathered}
\text { Free }_{\mathcal{F}_{m}^{f r}}\left(\partial\left(C[\ell+i, M] \times[0,1]^{i} / \Sigma_{i}\right) ; \ell\right) \longrightarrow \text { Free }_{\mathcal{F}_{m}^{f r}}\left(C[\ell+i, M] \times[0,1]^{i} / \Sigma_{i} ; \ell\right) \\
\downarrow \\
\widetilde{C}_{(\ell+i-1), i-1}^{f r}[\cdot, M] \longrightarrow \widetilde{C}_{(\ell+i-1), i}^{f r}[\cdot, M] .
\end{gathered}
$$

The truncated case follows from the fact that the generating strata of $\widetilde{C}_{(k)}^{f r}[\cdot, M]$ all lie in the components of degree less than or equal to $k$. 
Theorem 2.1 is a consequence of Propositions 2.4, 2.5, 2.6 and Lemma 2.7.

Proposition 2.5 For any right $\mathcal{F}_{m}$ module $\mathcal{N}(\cdot)$, the cofunctor that assigns to any $U \in \mathcal{O}(M)$ (respectively $U \in{ }^{\delta} \mathbb{M a n}_{m}$ ) the space

$$
\operatorname{Rmod}_{\mathcal{F}_{m}^{f r}}\left(\widetilde{C}_{(k)}^{f r}[\cdot, U], \mathcal{N}(\cdot)\right)
$$

is a homotopy $\mathcal{J}_{k}$-sheaf on $\mathcal{O}(M)$ (respectively ${ }^{\delta} \mathbb{M a n}_{m}$ ).

We prove this result in Section 3. The space (2-18) looks almost like the space of sections of a stratified fiber bundle over the filtered space

$$
\operatorname{Sub}_{\leq 0}(U) \subset \operatorname{Sub}_{\leq 1}(U) \subset \operatorname{Sub}_{\leq 2}(U) \subset \operatorname{Sub}_{\leq 3}(U) \subset \cdots \subset \operatorname{Sub}_{\leq k}(U) .
$$

Our argument is thus a slight adjustment of the proof of a similar result that the functor $U \mapsto \operatorname{Maps}\left(U^{\times k}, X\right)$ is polynomial of degree less than or equal to $k$; see [13, Proposition 3.1].

Proposition 2.6 The composition

$$
\begin{aligned}
\operatorname{Emb}(U, N) & \stackrel{\mathrm{ev}}{\rightarrow} \operatorname{Rmod}_{\mathcal{F}_{m}^{f r}}\left(C^{f r}[\cdot, U], C^{m_{-} f r}[\cdot, N]\right) \\
& \rightarrow \operatorname{Rmod}_{\mathcal{F}_{m}^{f r}}\left(\widetilde{C}_{(k)}^{f r}[\cdot, U], C^{m_{-} f r}[\cdot, N]\right)
\end{aligned}
$$

is a homotopy equivalence whenever $U$ is a disjoint union of less than or equal to $k$ $m$-balls.

The proof is given in Section 4.

Lemma 2.7 In case $M$ is orientable, respectively parallelized, one has a natural homeomorphism of spaces

$$
\operatorname{Rmod}_{\mathcal{F}_{m}^{f r}}\left(\widetilde{C}_{(k)}^{f r}[\cdot, M], C^{m_{-} f r}[\cdot, N]\right) \cong \operatorname{Rmod}_{\mathcal{F}_{m}^{o r}}\left(\widetilde{C}_{(k)}^{o r}[\cdot, M], C^{m \_f r}[\cdot, N]\right),
$$

respectively

$$
\operatorname{Rmod}_{\mathcal{F}_{m}^{f r}}\left(\widetilde{C}_{(k)}^{f r}[\cdot, M], C^{m_{-} f r}[\cdot, N]\right) \cong \operatorname{Rmod}_{\mathcal{F}_{m}}\left(\widetilde{C}_{(k)}[\cdot, M], C^{m_{-} f r}[\cdot, N]\right) .
$$

This lemma is obvious by inspection. 


\section{Proof of Proposition 2.5}

We need to show that the functor that assigns to any open set $U$ the space

$$
R_{k}(U, \mathcal{N}):=\operatorname{Rmod}_{\mathcal{F}_{m}^{f r}}\left(\widetilde{C}_{(k)}^{f r}[\cdot, U], \mathcal{N}(\cdot)\right)
$$

is a homotopy sheaf on $\mathcal{O}(M)$ with respect to the Grothendieck topology $\mathcal{J}_{k}$. This means that for any cover $\left\{U_{i} \subset U\right\}_{i \in I}$ such that $\bigcup_{i \in I} U_{i}^{\times k}=U^{\times k}$ one has that the natural map

$$
R_{k}(U, \mathcal{N}) \stackrel{\simeq}{\longrightarrow} \operatorname{holim}_{\varnothing \neq S \subset I} R_{k}\left(U_{S}, \mathcal{N}\right)
$$

is a weak equivalence. In the above the homotopy limit is taken over the category of finite nonempty subsets of $I$, and $U_{S}=\bigcap_{i \in S} U_{i}$.

Let us prove first that the functor

$$
U \mapsto \bar{R}_{k}(U, \mathcal{N}):=\operatorname{Rmod}_{\overline{\mathcal{F}}_{m}^{f r}}\left(C^{f r}[\cdot, U], \mathcal{N}(\cdot)\right)
$$

is polynomial of degree less than or equal to $k$. We don't need this result, but technically it is easier, and the proof of the statement that we need is just a slight modification of the argument given below.

For a set $J$ denote by $\Delta^{J}$ its formal convex hull. It consists of linear combinations $\vec{\lambda}=\sum_{i \in J} \lambda_{i}\langle i\rangle$ of elements in $J$, such that $\sum_{i \in J} \lambda_{i}=1 ; 0 \leq \lambda_{i} \leq 1, i \in J$; and the support of $\vec{\lambda}$ is finite:

$$
\operatorname{supp}(\vec{\lambda})=\left\{i \in J \mid \lambda_{i} \neq 0\right\}<\infty
$$

In case $J$ is finite $\Delta^{J}$ is a simplex. In the general case it is the realization of the full combinatorial simplicial complex on the vertex set $J$. In particular we have that $\Delta^{J}$ is naturally a CW-complex. The space holim $\varnothing \neq S \subset I \quad \bar{R}_{k}\left(U_{S}, \mathcal{N}\right)$ can be described as the space of natural transformations between the functor that assigns $\Delta^{S}$ to any finite nonempty set $S \subset I$ and the functor that assigns $\bar{R}_{k}\left(U_{S}, \mathcal{N}\right)$ to $S \subset I$. Thus a point $G$ in the homotopy limit is given by a family of maps

$$
G_{S}: \Delta^{S} \rightarrow \bar{R}_{k}\left(U_{S}, \mathcal{N}\right), \quad \varnothing \neq S \subset I .
$$

By adjunction this family of maps can be written as another collection of maps

$$
G_{S, k}: \Delta^{S} \times C^{f r}\left[k, U_{S}\right] \rightarrow \mathcal{N}(k), \quad \varnothing \neq S \subset I, \quad k \geq 0,
$$


that satisfy certain boundary conditions. In particular for $S_{1} \subset S_{2}$, one has $\Delta^{S_{1}} \subset \Delta^{S_{2}}$, $U_{S_{1}} \supset U_{S_{2}}$ and

$$
\left.G_{S_{2}, k}\right|_{\Delta} S_{1 \times C^{f r}\left[k, U_{S_{2}}\right]}=\left.G_{S_{1}, k}\right|_{\Delta S_{1 \times C^{f r}\left[k, U_{S_{2}}\right.}} .
$$

For this reason we drop the subindices $S$ and $k$ and will simply write $G(\vec{\lambda}, X)$, where $\vec{\lambda} \in \Delta^{I}$, and $X \in C^{f r}[k, U]$ for some $k \geq 0$. Notice that $G(\vec{\lambda}, X)$ is defined if and only if $g(X) \subset U_{\text {supp }(\vec{\lambda})}$.

One has a natural inclusion

$$
i: \bar{R}_{k}(U, \mathcal{N}) \longrightarrow \operatorname{holim}_{\varnothing \neq S \subset I} \bar{R}_{k}\left(U_{S}, \mathcal{N}\right)
$$

that sends $F$ to $(i F)(\vec{\lambda}, X)=F(X)$. Let us describe the homotopy inverse to $i$. Recall that $\operatorname{Sub}_{\leq k}(U)$. It is homeomorphic to a CW-complex and therefore is paracompact. One also has that $\operatorname{Sub}_{\leq k}\left(U_{i}\right), i \in I$, is an open cover of $\operatorname{Sub}_{\leq k}(U)$, since $\bigcup_{i \in I} U_{i}^{\times k}=$ $U^{\times k}$. Let $\vec{\psi}=\sum_{i \in I} \psi_{i}\langle i\rangle$ be a partition of unity on $\operatorname{Sub}_{\leq k}(U)$ subordinate to the above cover. We view it as a continuous map

$$
\vec{\psi}: \operatorname{Sub}_{\leq k}(U) \rightarrow \Delta^{I}
$$

that has the property $g \subset U_{\text {supp }}(\vec{\psi}(g))$ for any $g \in \operatorname{Sub}_{\leq k}(U)$. On the other hand, we also have that the map

$$
g: \coprod_{i=0}^{k} C^{f r}[i, U] \rightarrow \operatorname{Sub}_{\leq k}(U)
$$

is continuous. Now for $G \in \operatorname{holim}_{\varnothing \neq S \subset I} \bar{R}_{k}\left(U_{S}, \mathcal{N}\right)$ viewed as a function $G(\vec{\lambda}, X)$, we define $s(G) \in \bar{R}_{k}(U, \mathcal{N})$ by the formula

$$
s(G)(X)=G(\vec{\psi}(g(X)), X) .
$$

Since the right action of $\overline{\mathcal{F}}_{m}^{f r}$ preserves $g(X)$, see (2-5), we get that $s(G)$ is a morphism of right $\overline{\mathcal{F}}_{m}^{f r}$-modules. It is easy to see that $s \circ i$ is identity, whereas $i \circ s$ sends $G(\vec{\lambda}, X)$ to

$$
(i s)(G)(\vec{\lambda}, X)=G(\vec{\psi}(g(X)), X) .
$$

The homotopy between $G$ and $(i s)(G)$ is given by

$$
G(\tau \cdot \vec{\psi}(g(X))+(1-\tau) \cdot \vec{\lambda}, X), \quad 0 \leq \tau \leq 1 .
$$

Thus we proved that $\bar{R}_{k}(-, \mathcal{N})$ is a homotopy $\mathcal{J}_{k}$-sheaf. 
Now, let us establish the same result for $R_{k}(-, \mathcal{N})$. One still has an obvious map

$$
\begin{gathered}
i: R_{k}(U, \mathcal{N}) \longrightarrow \operatorname{holim}_{\varnothing \neq S \subset I} R_{k}\left(U_{S}, \mathcal{N}\right), \\
(i F)(\vec{\lambda}, \tilde{X})=F(\tilde{X}),
\end{gathered}
$$

where $\tilde{X} \in \widetilde{C}_{(k)}^{f r}[j, U], 0 \leq j \leq k$. For the homotopy inverse, unfortunately the formula (3-1) does not work since the analogous map

$$
\tilde{g}: \coprod_{i=0}^{k} \widetilde{C}_{(k)}^{f r}[i, U] \rightarrow \operatorname{Sub}_{\leq k}(U),
$$

that assigns the set of geometrically distinct points, is not continuous anymore. To remedy this we first introduce the map

$$
\Xi: \widetilde{C}^{f r}[\cdot, U] \rightarrow \widetilde{C}^{f r}[\cdot, U]
$$

that removes all hairs of $\tilde{X} \in \widetilde{C}^{f r}[k, U]$ of length $t \leq 1 / 2$, and contracts every hair to the length $2 t-1$ if its length $t \geq 1 / 2$. It is easy to see that $\Xi$ is an endomorphism of $\widetilde{C}^{f r}[k, U]$ as a right $\mathcal{F}_{m}^{f r}$-module. Moreover $\Xi$ preserves filtration (2-16) and is homotopic to the identity in the space of filtration preserving endomorphisms.

Now let $\tilde{X} \in \widetilde{C}^{f r}[k, U]$ have the form $\left(X ; y_{1} \cdots y_{\ell} ; t_{1} \cdots t_{\ell}\right)$, where $0<t_{1} \leq t_{2} \leq$ $\cdots \leq t_{h} \leq \frac{1}{2}<t_{h+1} \leq \cdots \leq t_{\ell} \leq 1$. Define $\tilde{g}_{i}(\tilde{X})=g(X) \cup\left\{y_{i+1}, y_{i+2}, \ldots, y_{\ell}\right\}$. One obviously has

$$
\tilde{g}(\tilde{X})=\tilde{g}_{0}(\tilde{X}) \supset \widetilde{g}_{1}(\tilde{X}) \supset \cdots \supset \widetilde{g}_{h}(\tilde{X})=\tilde{g}(\Xi(\tilde{X})) .
$$

Notice that $\widetilde{g}_{i}$ are not uniquely defined, and therefore are also discontinuous, in case $\tilde{X}$ has several hairs of the same length. For example if $t_{i}=t_{i+1}$ and all other $t_{j}$ are different there is a choice which hair we take as the $i^{\text {th }}$ one and which we take as the $(i+1)$-st. Thus in this particular case the set $\tilde{g}_{i}(\tilde{X})$ is not uniquely defined, but however all the other sets including $\tilde{g}_{i-1}(\tilde{X})$ are defined uniquely. Now define a map

$$
\begin{gathered}
\vec{\phi}: \coprod_{i=0}^{k} \widetilde{C}_{(k)}^{f r}[i, U] \rightarrow \Delta^{I}, \\
\vec{\phi}(\tilde{X})=\sum_{i=0}^{h} 2\left(t_{i+1}-t_{i}\right) \vec{\psi}\left(\widetilde{g}_{i}(\tilde{X})\right),
\end{gathered}
$$


where $t_{0}=0$ and abusing notation $t_{h+1}=1 / 2$. Thus the sum of coefficients $\sum_{i=0}^{h} 2\left(t_{i+1}-t_{i}\right)=1$. We argue below that $\vec{\phi}$ is continuous. Recall (2-7):

$$
\widetilde{C}_{(k)}^{f r}[i, U]=\left(\coprod_{j=0}^{k-i} C^{f r}[i, U] \times\left(U^{\times j} \times[0,1]^{j}\right) / \Sigma_{j}\right) / \sim .
$$

Thus we are left to check that the equivalence relations (2-8), (2-9) and (2-10) are respected by $\vec{\phi}$, which is an easy exercise.

Now we are ready to define a map $s$ homotopy inverse to (3-2):

$$
(s G)(\tilde{X})=G(\vec{\phi}(\tilde{X}), \Xi(\tilde{X})) .
$$

This formula is well-defined since $\tilde{g}(\Xi(\tilde{X})) \subset U_{\text {supp }(\vec{\phi}(\tilde{X})) \text {. Indeed, we have that }}$ $\tilde{g}(\Xi(\tilde{X}))=\widetilde{g}_{h}(\tilde{X}) \subset \tilde{g}_{i}(\tilde{X}) \subset U_{\operatorname{supp}}\left(\vec{\psi}\left(\tilde{g}_{i}(\tilde{X})\right)\right)$, for $0 \leq i \leq h$. On the other hand, we have $\bigcap_{i=0}^{h} U_{\text {supp }}\left(\vec{\psi}\left(\tilde{g}_{i}(\tilde{X})\right)\right) \subset U_{\operatorname{supp}(\vec{\phi}(\tilde{X}))}$.

One also has that $s G$ is a morphism of right modules over $\mathcal{F}_{m}^{f r}$ since the right $\mathcal{F}_{m}^{f r}$ action does not change $\vec{\phi}(\tilde{X})$ :

$$
\vec{\phi}\left(\tilde{X} \circ_{i} c\right)=\vec{\phi}(\tilde{X})
$$

for every $c \in \mathcal{F}_{m}^{f r}(\cdot)$, since again each $\widetilde{g}_{i}$ is preserved by this action.

We check that $s$ is a homotopy inverse to $i$. One has $(s i)(F)=F \circ \Xi$. Since $\Xi$ is homotopic to the identity $(s i)$ is so. For the opposite composition we have

$$
(i s)(G)(\vec{\lambda}, \tilde{X})=G(\vec{\phi}(\tilde{X}), \Xi(\tilde{X})) .
$$

The homotopy

$$
G(\tau \cdot \vec{\lambda}+(1-\tau) \cdot \vec{\phi}(\tilde{X}), \Xi(\tilde{X})), \quad 0 \leq \tau \leq 1,
$$

shows that $(i s)$ is homotopic to the map that sends $G(\vec{\lambda}, \tilde{X})$ to $H(\vec{\lambda}, \tilde{X})=G(\vec{\lambda}, \Xi(\tilde{X}))$. Finally using the homotopy between $\Xi$ and the identity we see that $(i s)$ is also homotopic to the identity.

This finishes the proof of Proposition 2.5.

\section{Proof of Proposition 2.6}

We need to show that the natural evaluation map

$$
\operatorname{ev}_{k}: \operatorname{Emb}(U, N) \rightarrow \operatorname{Rmod}_{\mathcal{F}_{m}^{f r}}\left(\widetilde{C}_{(k)}^{f r}[\cdot, U], C^{m_{-} f r}[\cdot, N]\right)
$$


is a homotopy equivalence whenever $U$ is a disjoint union of $\ell$ balls with $\ell \leq k$. Let $L \subset U$ be a finite subset of $U$ with exactly one point in each connected component. We fix a bijection $b:\{1 \cdots \ell\} \rightarrow L$ and also framings $\alpha_{i}: \mathbb{R}^{m} \rightarrow T_{b(i)} U$ for each point in $L$. We denote by $L^{f r}$ the corresponding point in $C^{f r}(\ell, M)$. One has a natural evaluation map

$$
\operatorname{Ev}_{L^{f r}}: \operatorname{Emb}(U, N) \stackrel{\simeq}{\longrightarrow} C^{m_{-} f r}(L, N),
$$

that sends $f \in \operatorname{Emb}(M, N)$ to the configuration $f(b(1)) \cdots f(b(\ell))$ with framings defined as compositions

$$
\mathbb{R}^{m} \stackrel{\alpha_{i}}{\longrightarrow} T_{b(i)} U \stackrel{f_{*}}{\longrightarrow} T_{f(b(i))} N .
$$

One can easily see that this map is a homotopy equivalence; see for example [13].

Let $C^{f r}[\cdot, L]$ denote the right $\mathcal{F}_{m}^{f r}$ submodule of $\widetilde{C}^{f r}[\cdot, U]$ which is generated by $L^{f r} \in C^{f r}[\ell, L]$. It is easy to see that this submodule is naturally homeomorphic to a free right module generated by $\Sigma_{\ell}$ in degree $\ell$. In other words the map of right $\mathcal{F}_{m}^{f r}$ modules

$$
\text { Free }_{\mathcal{F}_{m}^{f r}}^{f(}\left(\Sigma_{\ell}, \ell\right) \rightarrow C^{f r}[\cdot, L]
$$

that sends the unit of $\Sigma_{\ell}$ to $L^{f r}$, is a homeomorphism. Therefore the $k^{\text {th }}$ truncation $C^{f r}\left[\left.\cdot\right|_{\leq k}, L\right]$ is also a truncated right module freely generated by $\Sigma_{\ell}$ in degree $\ell$ (here we use the fact that $\ell \leq k$ ).

The inclusion

$$
C^{f r}[\cdot, L] \subset \widetilde{C}^{f r}[\cdot, U]
$$

is a homotopy equivalence of right $\mathcal{F}_{m}^{f r}$ modules. The homotopy inverse is obtained by contracting each disc in $U$ to the corresponding point in $L$, thus sending usual configurations to infinitesimal configurations. Notice that the same is true for the inclusion of the truncations

$$
C^{f r}\left[\left.\cdot\right|_{\leq k}, L\right] \subset \widetilde{C}_{(k)}^{f r}\left[\left.\cdot\right|_{\leq k}, U\right] .
$$

As a result we get a sequence of homotopy equivalences

$$
\begin{array}{r}
\operatorname{Rmod}_{\mathcal{F}_{m}^{f r}}\left(\widetilde{C}_{(k)}^{f r}[\cdot, U], C^{m_{-} f r}[\cdot, N]\right) \stackrel{\simeq}{\longrightarrow} \operatorname{Rmod}_{\mathcal{F}_{m}^{f r}}\left(C^{f r}[\cdot, L], C^{m \_f r}[\cdot, N]\right) \\
\stackrel{\cong}{\longrightarrow} \operatorname{Maps}_{\Sigma_{\ell}}\left(\Sigma_{\ell}, C^{m_{-} f r}[\ell, N]\right) \stackrel{\cong}{\longrightarrow} C^{m_{-} f r}[\ell, N] .
\end{array}
$$


The last two maps in (4-1) are homeomorphisms. Finally we notice that the diagram

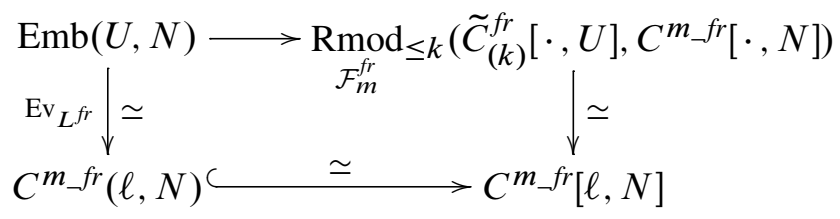

is commutative (the right arrow is the composition (4-1)). We conclude that the top arrow must be an equivalence since all the other maps are.

\section{Proof of Theorem 1.5 and Remark 2.2}

\subsection{Theorem 1.5}

By the universal property of polynomial functors [25] one only needs to show that the functor

$$
U \mapsto \operatorname{hRmod}_{\mathcal{E}\left(D^{m}\right)}(\operatorname{Emb}(\cdot, U), F(\cdot)), \quad U \in{ }^{\delta} \operatorname{Man}_{m},
$$

is polynomial of degree less than or equal to $k$, and also that for every $U$ which is a disjoint union of less than or equal to $k$ balls this functor produces a space naturally equivalent to $F(U)$. Notice that the cofunctor $\operatorname{Emb}(\cdot, U)$, where $U \in \mathcal{O}_{\leq k}$, is representable and therefore cofibrant; thus the latter statement is straightforward from the Yoneda lemma and also the fact that the category of $k$-truncated right modules over $\mathcal{E}\left(D^{m}\right)$ is equivalent to the category of contravariant functors from the category $\mathcal{O}_{\leq k}$. Let $W\left(\mathcal{E}\left(D^{m}\right)\right)$ be the Boardmann-Vogt replacement of the operad $\mathcal{E}\left(D^{m}\right)$, see [4]; and let $W(-)$ be a similar Boardmann-Vogt resolution of the corresponding right module over $\mathcal{E}\left(D^{m}\right)$. Due to the zigzag of equivalences of operads [18], we have

$$
\mathcal{E}\left(D^{m}\right) \longleftarrow W\left(\mathcal{E}\left(D^{m}\right)\right) \longrightarrow \mathcal{F}_{m}^{f r}
$$

and the zigzag of right modules over $W\left(\mathcal{E}\left(D^{m}\right)\right)$

$$
\operatorname{Emb}(\cdot, U) \longleftarrow W(\operatorname{Emb}(\cdot, U)) \longrightarrow C^{f r}[\cdot, U]
$$

which is natural in $U$, the functor (5-1) is equivalent to a similar functor

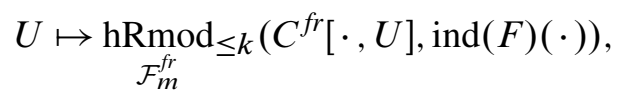


where ind $(F)$ is a certain right $\mathcal{F}_{m}^{f r}$ module obtained from $F(\cdot)$ by a natural restrictionextension construction along the zigzag (5-2); see Fresse [7, Theorem 16.B]. By Proposition 2.5 the functor (5-4) is polynomial of degree less than or equal to $k$, therefore the equivalent functor (5-1) is so.

\subsection{Remark 2.2}

We need to show that the cofunctors

$$
\begin{aligned}
& U \mapsto \underset{\mathcal{E}\left(D^{m}\right)}{\operatorname{hRmod}_{\leq k}(\operatorname{Emb}(\cdot, U), \operatorname{Emb}(\cdot, N)),} \quad U \in{ }^{\delta} \operatorname{Man}_{m}, \\
& U \mapsto \underset{\mathcal{F}_{m}^{f r}}{\mathrm{hRmod}_{\leq k}\left(C^{f r}[\cdot, U], C^{m_{-} f r}[\cdot, N]\right),} \quad U \in{ }^{\delta} \mathbb{M a n}_{m},
\end{aligned}
$$

are equivalent. To see this equivalence we recall that the operad $\mathcal{F}_{m}^{f r}$ is equivalent to $\mathcal{E}\left(D^{m}\right)$ via the zigzag (5-2). Thus by [7, Theorem 16.B] the right-hand sides of (5-5) and (5-6) can be expressed as spaces of derived maps of (truncated) right modules over $W\left(\mathcal{E}\left(D^{m}\right)\right)$. Finally, one has a similar zigzag of equivalences of right $W\left(\mathcal{E}\left(D^{m}\right)\right)$-modules

$$
\operatorname{Emb}(\cdot, N) \longleftarrow W(\operatorname{Emb}(\cdot, N)) \longrightarrow C^{m_{-} f r}[\cdot, N],
$$

which together with the natural in $U \in{ }^{\delta} \mathbb{M a n}_{m}$ zigzag (5-3) imply Remark 2.2.

\section{Spaces of long embeddings}

Theorem 1.5 has a particularly attractive form for the spaces of higher dimensional long knots. Let $\overline{\operatorname{Emb}}_{c}\left(\mathbb{R}^{m}, \mathbb{R}^{n}\right)$ denote the homotopy fiber of the inclusion

$$
\operatorname{Emb}_{c}\left(\mathbb{R}^{m}, \mathbb{R}^{n}\right) \hookrightarrow \operatorname{Imm}_{c}\left(\mathbb{R}^{m}, \mathbb{R}^{n}\right),
$$

where $\operatorname{Emb}_{c}\left(\mathbb{R}^{m}, \mathbb{R}^{n}\right)$, and respectively $\operatorname{Imm}_{c}\left(\mathbb{R}^{m}, \mathbb{R}^{n}\right)$, is the space of embeddings $\mathbb{R}^{m} \hookrightarrow \mathbb{R}^{n}$, respectively immersions $\mathbb{R}^{m} \rightarrow \mathbb{R}^{n}$, coinciding with a fixed linear embedding $i: \mathbb{R}^{m} \hookrightarrow \mathbb{R}^{n}$ outside a compact subset of $\mathbb{R}^{m}$. We view $\overline{\operatorname{Emb}}_{c}\left(-, \mathbb{R}^{N}\right)$ as a cofunctor $\widetilde{\mathcal{O}}\left(\mathbb{R}^{m}\right) \rightarrow$ Top from the category of open sets of $\mathbb{R}^{m}$ whose complement is compact. Define $\widetilde{\mathcal{O}}_{\leq k}\left(\mathbb{R}^{m}\right)$ as its full subcategory that consists of disjoint unions of less than or equal to $k$ balls and one complement to a closed ball. For an isotopy invariant cofunctor $F: \widetilde{\mathcal{O}}\left(\mathbb{R}^{m}\right) \rightarrow$ Top, its $k^{\text {th }}$ Taylor approximation $T_{k} F$ is the homotopy right Kan extension of $F$ from $\widetilde{\mathcal{O}}_{\leq k}\left(\mathbb{R}^{m}\right)$ to $\widetilde{\mathcal{O}}\left(\mathbb{R}^{m}\right)$. 
To take into account the behavior of embeddings at infinity, we will express spaces of such embeddings as spaces of derived morphisms of certain infinitesimal bimodules over the little discs operad; see Loday and Vallette [14]. ${ }^{3}$ An infinitesimal bimodule over an operad is defined in the following way. Let $\{O(i), i \geq 0\}$ be an operad. An infinitesimal bimodule over $O$ is a symmetric sequence $\{M(i), i \geq 0\}$ equipped with structure maps (where $i \geq s \geq 1, j \geq 0$, and $\otimes$ stands for a symmetric monoidal product):

$$
\begin{array}{cc}
\circ_{s}: O(i) \otimes M(j) \longrightarrow M(i+j-1) & \text { left action, } \\
\mathrm{o}_{s}^{\prime}: M(i) \otimes O(j) \longrightarrow M(i+j-1) & \text { right action, }
\end{array}
$$

satisfying certain rather easily guessed associativity axioms [2]. For example, left and right actions must be compatible:

$$
\begin{array}{ll}
\left(o_{1} \circ_{p} m\right) \circ_{q} o_{2}=\left(o_{1} \circ_{q} o_{2}\right) \circ_{p+q-1} m, & 1 \leq q<p \leq i, \\
\left(o_{1} \circ_{p} m\right) \circ_{q+k-1} o_{2}=\left(o_{1} \circ_{q} o_{2}\right) \circ_{p} m, & 1 \leq p<q \leq i,
\end{array}
$$

for all $o_{1} \in O(i), o_{2} \in O(j)$, and $m \in M(k)$. As above the result of composition $\circ_{i}(o, m)$, and $\circ_{i}^{\prime}(m, o)$, for $o \in O(n)$, and $m \in M(k)$, is denoted by $o \circ_{i} m$, and $m \circ_{i} o$. Graphically one can view elements of $O$ and $M$ as having a bunch of inputs and one output. In this representation the composition is shown by the usual grafting; see Figure 1.

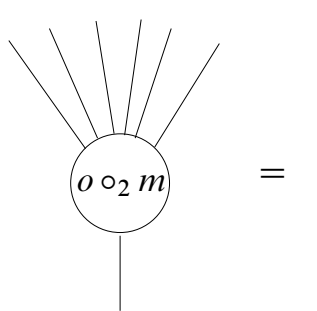

Left action

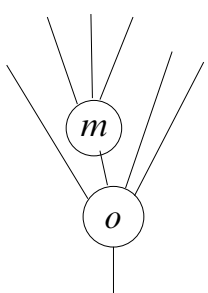

Figure 1: Infinitesimal action

Notice that this structure is different from the structure of a bimodule over an operad. Moreover neither structure can be obtained from one another. Indeed, the right infinitesimal action is the same as the usual right action, but the left infinitesimal action is different from the usual left action. However, in one important case when a bimodule $M$ is endowed with a morphism (of bimodules over $\mathcal{O}$ ) $\mathcal{O} \rightarrow M$, the sequence $M$ inherits the structure of an infinitesimal bimodule. Indeed the image of

\footnotetext{
${ }^{3}$ In some previous works the author was using the term weak bimodules for this notion as for example in [23].
} 
the unit of $\mathcal{O}$ in $M$ can be used to mimic the empty insertions of the left action. In particular if $\mathcal{O} \rightarrow \mathcal{P}$ is a morphism of operads, then $\mathcal{P}$ is naturally an infinitesimal bimodule over $\mathcal{O}$. As an example relevant to us, the linear inclusion $i: \mathbb{R}^{m} \hookrightarrow \mathbb{R}^{n}$ induces an inclusion of operads of little discs $\mathcal{B}_{m} \hookrightarrow \mathcal{B}_{n}$, and thus both $\mathcal{B}_{m}$ and $\mathcal{B}_{n}$ are infinitesimal bimodules over $\mathcal{B}_{m}$.

As another example an infinitesimal bimodule over the commutative operad is the same thing as a contravariant functor from the category $\Gamma$ of finite pointed sets. An infinitesimal bimodule over the non $-\Sigma$ associative operad is nothing but a cosimplicial object.

By a $k$-truncated infinitesimal bimodule over $O$ we will understand a symmetric sequence $\{M(i), i=0, \ldots, k\}$, with the above structure maps in the range where they can be defined.

The category of (truncated) infinitesimal bimodules has all the pleasant formal properties of right modules. For example, the category of infinitesimal bimodules with values in chain complexes is (in contrast with the category of honest left modules) an abelian category with enough projectives. Another nice property of this structure is that an equivalence of operads induces restriction and extension functors which are Quillen equivalences, similarly to the case of right modules [7, Theorem 16.B]. Let hIbimod $(-,-), \operatorname{hIbimod}_{\leq k}(-,-)$ denote the space of derived morphisms between infinitesimal bimodules and $k$-truncated infinitesimal bimodules respectively.

\section{Theorem 6.1 One has natural equivalences}

$$
T_{k} \overline{\operatorname{Emb}}_{c}\left(\mathbb{R}^{m}, \mathbb{R}^{n}\right) \simeq \operatorname{hIbimod}_{\mathcal{B}_{m}}\left(\mathcal{B}_{m}, \mathcal{B}_{n}\right), \quad n \geq m .
$$

In particular in the case $n>m+2$,

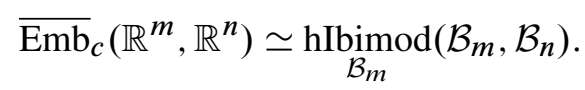

A discrete version of this theorem appeared in [2]. In the last statement of the theorem we use the unpublished result of Goodwillie, Klein, and Weiss about the convergence of the embedding tower in codimension greater than or equal to 3 . This statement generalizes Sinha's production [20] of a cosimplicial space $\mathcal{K}_{n}^{\bullet}$ whose homotopy totalization Tot $\mathcal{K}_{n}^{\bullet}$ is weakly equivalent to $\overline{\operatorname{Emb}}_{c}\left(\mathbb{R}^{1}, \mathbb{R}^{n}\right), n \geq 4$. This cosimplicial object arises from an operad $\mathcal{K}_{n}$ equipped with a map $\mathcal{A s s o c} \rightarrow \mathcal{K}_{n}$, where $\mathcal{A s s o c}$ is the associative operad (which is equivalent to $\mathcal{B}_{1}$ ), $\mathcal{K}_{n}$ is an operad equivalent to $\mathcal{B}_{n}$, and the map in question is equivalent to the usual inclusion $\mathcal{B}_{1} \rightarrow \mathcal{B}_{n}$. As was already mentioned, a cosimplicial space amounts exactly to an infinitesimal bimodule 
over Assoc in the category of spaces, and for $m=1$ our theorem above is the same as Sinha's formula:

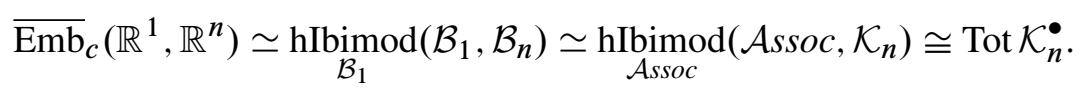

Theorem 6.1 follows from Theorem 6.3 and Lemma 6.2, and also from the fact that the inclusion of operads $\mathcal{B}_{m} \hookrightarrow \mathcal{B}_{n}$ is equivalent to the inclusion $\mathcal{F}_{m} \hookrightarrow \mathcal{F}_{n}$, which means that there is a zigzag of morphisms of operads in which every horizontal arrow is an equivalence:

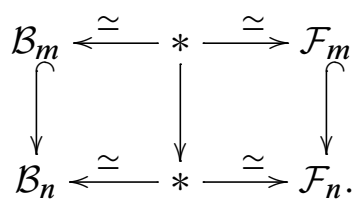

In fact the middle map can be chosen to be $W\left(\mathcal{B}_{m}\right) \hookrightarrow W\left(\mathcal{B}_{n}\right)$; see [18].

Consider the sequence

$$
C_{*}\left[\cdot, S^{n}\right]=\left\{C_{*}\left[k, S^{n}\right], k \geq 0\right\}
$$

where $C_{*}\left[k, S^{n}\right]$ is the Fulton-MacPherson-Axelrod-Singer compactification of the configuration space of $k+1$ distinct points in $S^{n}$ labeled by $\{*, 1,2, \ldots, k\}$, one of which, labeled by $*$, is fixed to be $\infty \in S^{n}$. Here and below we view $S^{n}$ as a one-point compactification of $\mathbb{R}^{n}$. Thus the interior of $C_{*}\left[k, S^{n}\right]$ is naturally identified with the configuration space $C\left(k, \mathbb{R}^{n}\right)$. It turns out that $C_{*}\left[\cdot, S^{n}\right]$ is naturally an infinitesimal bimodule over $\mathcal{F}_{n}$ (and therefore over $\mathcal{F}_{m}, m \leq n$ ). Let $p_{i}: C_{*}\left[k, S^{n}\right] \rightarrow S^{n}$, $i=1, \ldots, k$, be natural projections. To define a right $\mathcal{F}_{n}$ action on this sequence we fix a framing of each projection $p_{i}(X) \in S^{n}, i=1, \ldots, k, X \in C_{*}\left[k, S^{n}\right]$. In case $p_{i}(X) \in \mathbb{R}^{n}$, the framing $\alpha_{i}: \mathbb{R}^{n} \rightarrow T_{p_{i}(X)} S^{n}$ is fixed to be the natural identification $\mathbb{R}^{n} \cong T_{p_{i}(X)} \mathbb{R}^{n} \cong T_{p_{i}(X)} S^{n}$. For the points $X$ on the boundary of $C_{*}\left[k, S^{n}\right]$ the framing is extended by continuity. It is quite easy to see that in case $p_{i}(X)=\infty$ this framing of $p_{i}(X)$ depends only on the direction from which $p_{i}(X)$ approaches $*$. Those framings enable $C_{*}\left[\cdot, S^{n}\right]$ with a right $\mathcal{F}_{n}$ action. Indeed, the right action of $\mathcal{F}_{n}$ replaces the corresponding point in the configuration $X$ by an infinitesimal configuration $c \in \mathcal{F}_{n}(\cdot)$ inserted according to the framing. The infinitesimal left action produces so called "strata at infinity". Let $s$ be the inversion map considered as a coordinate chart at $\infty$ :

$$
s:\{\infty\} \cup \bigcup\left(\mathbb{R}^{n} \backslash\{0\}\right) \longrightarrow \mathbb{R}^{n}, \quad x \mapsto x /|x|^{2}
$$


Define the framing at $*$ to be the natural identification $\mathbb{R}^{n} \cong T_{0} \mathbb{R}^{n}=T_{s(\infty)} \mathbb{R}^{n} \cong T_{\infty} S^{n}$. The left action $c \circ_{i} X$, where $c \in \mathcal{F}_{n}(\ell)$, replaces the point $*$ by an infinitesimal configuration $s_{i}(c)$, where

$$
s_{i}: \mathcal{F}_{n} \rightarrow \mathcal{F}_{n}
$$

is the inversion with the center $i^{\text {th }}$ point. The replacement is done according to the framing at infinity that we described above.

The reader might have an impression that this construction is more difficult than it actually is. It might appear difficult only because when points escape to infinity we describe how the situation looks like from the point of view of $\infty \in S^{n}$. But if we always keep the global picture in mind from the point of view of the observer in $\mathbb{R}^{n}$ then we can see that there is no twist in the framing and everything remains as flat as $\mathbb{R}^{n}$ is.

To be precise there exist two ways to describe $C_{*}\left[k, S^{n}\right]$ and its strata. The first one is the usual one (see [19]) which we call spherical, and which was used above to describe the infinitesimal action of $\mathcal{F}_{n}$ on $C_{*}\left[\cdot, S^{n}\right]$. In the second description, that we call flat, it is much easier to see that $C_{*}\left[\cdot, S^{n}\right]$ is naturally an infinitesimal bimodule over $\mathcal{F}_{n}$. The difference is that in the spherical model we look at how points approach $*=\infty$ in $S^{n}$, while in the flat model we look at how points escaping to infinity are located with respect to one another in $\mathbb{R}^{n}$. For example, consider the situation when points 3,4 , and 5, remain fixed in $\mathbb{R}^{n}$, and points 1 and 2 escape to infinity. The corresponding stratum in $C_{*}\left[5, S^{n}\right]$ is the product $C\left(3, \mathbb{R}^{n}\right) \times C\left(3, \mathbb{R}^{n}\right) / G$, where $G$ is the group of translations and positive rescalings. In the flat model, the first factor describes the location of 3, 4, 5 in $\mathbb{R}^{n}$; the second factor describes the relative location of 1,2 , and conglomeration $x=\{3,4,5\}$. Figure 2 represents a point in such stratum.

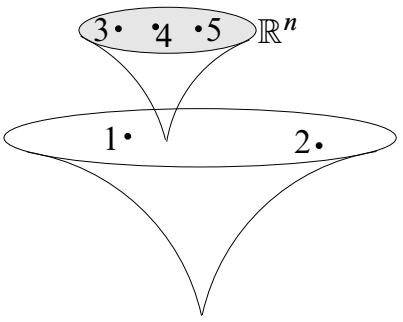

Figure 2

The upper disc describes the actual world $\mathbb{R}^{n}$ (it is not quotiented out by $G$ ); the lower disc describes how points escape to infinity. In particular we see that 2 escapes to infinity approximately 5 times faster than 1 . However, from the perspective of the point 
at infinity the picture is different; see Figure 3 . Since 2 escapes to $\infty$ faster, it is closer to $*$ than 1 . The configuration of points $1,2, *$ at infinity is obtained from the "flat configuration" of $1,2, x=\{3,4,5\}$, by taking inversion with center $x$.

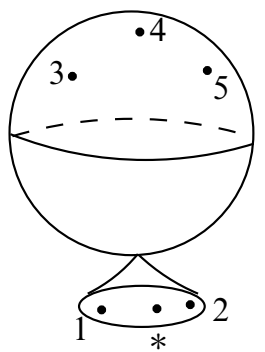

Figure 3

As a more general example, consider the stratum of $C_{*}\left[8, S^{n}\right]$ encoded by the tree from Figure 4. For the configurations in this stratum, points $6,7,8$ collide together; similarly 3 and 5 collide; points 1,4 , and 2 escape to infinity, but while doing so 1 stays close to 4 .
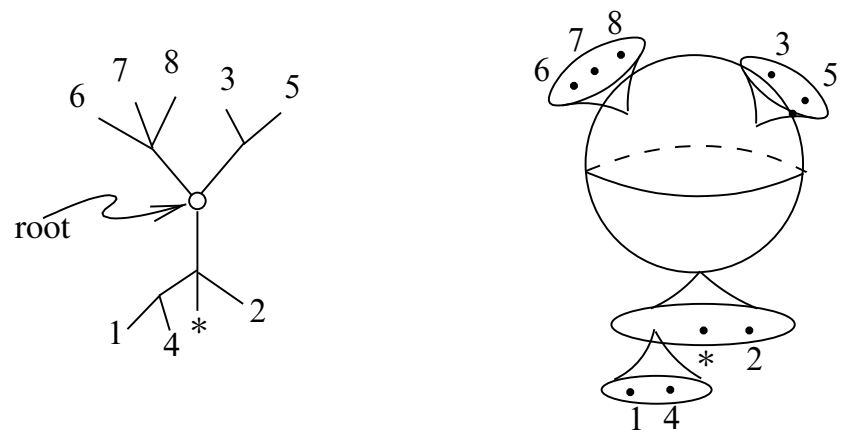

Figure 4

Figure 5 describes the corresponding limiting configuration in the flat model, in which we look at how the points are located with respect to one another, rather than at how they are located with respect to $\infty$. The shaded disc in the above figure is the actual world $\mathbb{R}^{n}$.

Both flat and spherical models parametrize their strata as products

$$
C\left(|r|, \mathbb{R}^{n}\right) \times \prod_{v \in V(T)} C\left(|v|, \mathbb{R}^{n}\right) / G
$$

where $T$ is a tree encoding the stratum, $r$ is its root, $V(T)$ is its set of nonroot-nonleaf vertices, $|v|$ is the valence of $v$ minus 1 . To pass from the spherical parametrization 

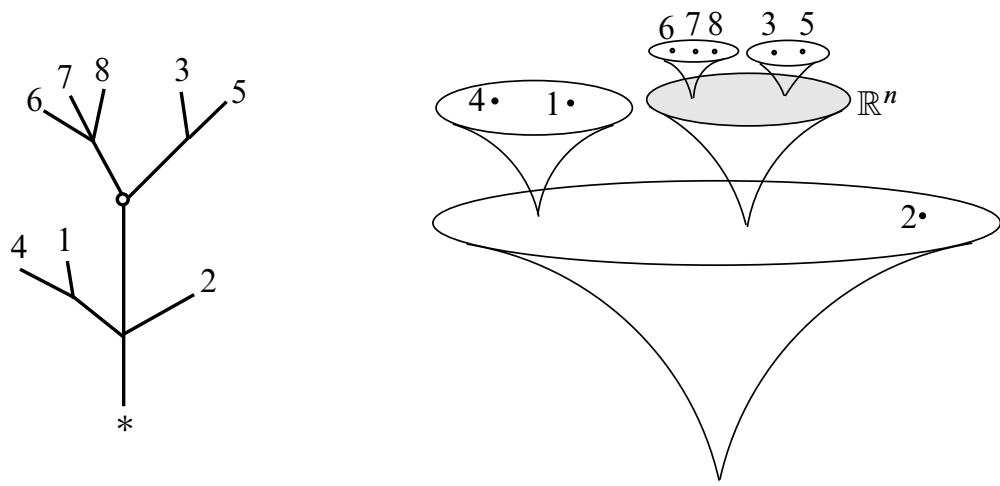

Figure 5

of a stratum encoded by a tree $T$ to the flat one, one needs to take inversion of the factors $C\left(|v|, \mathbb{R}^{n}\right) / G$ that correspond to the vertices $v$ lying on the path between $*$ and the root $r$ in $T$. For all the other factors, the map that gives correspondence is either identity in case the vertex is above the root or a framing adjusting rotation in case the vertex is below the root.

We mention that the flat description of $C_{*}\left[k, S^{n}\right]$ is alluded in the Bott-Taubes integration when one considers "strata at infinity", as for example by Cattaneo, Cotta-Ramusino and Longoni in [6].

To recall both $C_{*}\left[k, S^{n}\right]$ and $\mathcal{F}_{n}(k)$ are manifolds with corners whose interiors are respectively $C\left(k, \mathbb{R}^{n}\right)$ and $C\left(k, \mathbb{R}^{n}\right) / G$.

Lemma 6.2 The projection $C\left(k, \mathbb{R}^{n}\right) \rightarrow C\left(k, \mathbb{R}^{n}\right) / G$, where $G$ is the group of translations and positive rescalings, induces a continuous map $C_{*}\left[k, S^{n}\right] \rightarrow \mathcal{F}_{n}(k)$, $k \geq 0$, which defines an equivalence of infinitesimal $\mathcal{F}_{n}$ bimodules $C_{*}\left[\cdot, S^{n}\right] \rightarrow F_{n}(\cdot)$.

We skip the proof of this lemma. The most difficult part of the proof is probably checking that the induced map is a morphism of infinitesimal bimodules, which is however straightforward from the flat description of $C_{*}\left[k, S^{n}\right]$.

Theorem 6.3 For all $k \geq 0$ and all $n \geq m$ one has an equivalence

$$
T_{k} \overline{\operatorname{Emb}}_{c}\left(\mathbb{R}^{m}, \mathbb{R}^{n}\right) \simeq \operatorname{hIbimod}_{\mathcal{F}_{m}}\left(C_{*}\left[\cdot, S^{m}\right], C_{*}\left[\cdot, S^{n}\right]\right) .
$$

This theorem is a consequence of Proposition 6.4 and Theorem 6.5 below.

Notice that we are in a similar situation as in Section 2: one can see that $C_{*}\left[\cdot, S^{m}\right]$ is cofibrant as an infinitesimal bimodule over the reduced Fulton-MacPherson operad $\overline{\mathcal{F}}_{m}$. 
Thus one only needs to correct it a little bit in order to make the (right) action of $\mathcal{F}_{m}(0)$ to be free. Define $\widetilde{C}_{*}\left[\cdot, S^{m}\right]$ as the sequence of spaces

$$
\widetilde{C}_{*}\left[k, S^{m}\right]=\left(\coprod_{\ell \geq 0} \tilde{C}_{*}\left[k, S^{m}\right] \times\left(\left(S^{m}\right)^{\times \ell} \times[0,1]^{\ell}\right) / \Sigma_{\ell}\right) / \sim
$$

of hairy configurations. The equivalence relations are (2-8), (2-9), (2-10), plus in addition (2-10) must hold whenever $y_{i}=\infty$. In words the last condition says that a hair must disappear whenever it approaches infinity.

Define a filtration

$$
\widetilde{C}_{*}^{(0)}\left[\cdot, S^{m}\right] \subset \widetilde{C}_{*}^{(1)}\left[\cdot, S^{m}\right] \subset \widetilde{C}_{*}^{(2)}\left[\cdot, S^{m}\right] \subset \cdots
$$

similar to (2-16) by the number of geometrically distinct points different from $\infty$.

Proposition 6.4 The natural projection

$$
\widetilde{C}_{*}^{(k)}\left[\left.\cdot\right|_{\leq k}, S^{m}\right] \rightarrow C_{*}\left[\left.\cdot\right|_{\leq k}, S^{m}\right]
$$

is a cofibrant replacement of $k$-truncated infinitesimal bimodules over $\mathcal{F}_{m}$.

The proof is similar to Proposition 2.4.

Theorem 6.5 For $n \geq m$ and any $k \geq 0$ one has

$$
T_{k} \overline{\operatorname{Emb}}_{c}\left(\mathbb{R}^{m}, \mathbb{R}^{n}\right) \simeq \operatorname{Ibimod}_{\mathcal{F}_{m}}\left(\widetilde{C}_{*}^{(k)}\left[\cdot, S^{m}\right], C_{*}\left[\cdot, S^{n}\right]\right) .
$$

In the above Ibimod $\leq k$ denotes the space of (nonderived) morphisms of truncated infinitesimal bimodules. The main idea is that for any $U \in \widetilde{\mathcal{O}}\left(\mathbb{R}^{m}\right)$ one can similarly define infinitesimal bimodules $\widetilde{C}_{*}[\cdot, U]$ and then one can prove statements similar to Proposition 2.5 and Proposition 2.6 which imply the result.

Acknowledgements The author is grateful to G Arone, P Boavida de Brito, B Fresse, J Hughes, M Kontsevich, P Lambrechts, P Salvatore, and B Vallette for discussions and communication. Finally the author is thankful to the referee for a careful reading and useful corrections.

This work was supported by the NSF grant DMS 0968046. 


\section{References}

[1] G Arone, Derivatives of embedding functors, I, The stable case, J. Topol. 2 (2009) 461-516 MR2546583

[2] G Arone, V Turchin, On the rational homology of high dimensional analogues of spaces of long knots arXiv:1105.1576v2

[3] S Axelrod, I M Singer, Chern-Simons perturbation theory, II, J. Differential Geom. 39 (1994) 173-213 MR1258919

[4] J M Boardman, R M Vogt, Homotopy invariant algebraic structures on topological spaces, Lecture Notes in Mathematics 347, Springer, Berlin (1973) MR0420609

[5] P Boavida de Brito, MS Weiss, Manifold calculus and homotopy sheaves arXiv: 1202.1305

[6] A S Cattaneo, P Cotta-Ramusino, R Longoni, Configuration spaces and Vassiliev classes in any dimension, Algebr. Geom. Topol. 2 (2002) 949-1000 MR1936977

[7] B Fresse, Modules over operads and functors, Lecture Notes in Mathematics 1967, Springer, Berlin (2009) MR2494775

[8] E M Friedlander, G Mislin, Cohomology of classifying spaces of complex Lie groups and related discrete groups, Comment. Math. Helv. 59 (1984) 347-361 MR761803

[9] E Getzler, J D S Jones, Operads, homotopy algebra and iterated integrals for double loop spaces arXiv:hep-th/9403055

[10] T G Goodwillie, Calculus, I, The first derivative of pseudoisotopy theory, $K$-Theory 4 (1990) 1-27 MR1076523

[11] T G Goodwillie, Calculus, II, Analytic functors, $K$-Theory 5 (1991/92) 295-332 MR1162445

[12] T G Goodwillie, Calculus, III, Taylor series, Geom. Topol. 7 (2003) 645-711 MR2026544

[13] T G Goodwillie, J R Klein, MS Weiss, A Haefliger style description of the embedding calculus tower, Topology 42 (2003) 509-524 MR1953238

[14] J-L Loday, B Vallette, Algebraic operads, Grundl. Math. Wissen. 346, Springer, Heidelberg (2012) MR2954392

[15] J Milnor, On the homology of Lie groups made discrete, Comment. Math. Helv. 58 (1983) 72-85 MR699007

[16] F Morel, On the Friedlander-Milnor conjecture for groups of small rank, from: "Current developments in mathematics, 2010", (D Jerison, B Mazur, T Mrowka, W Schmid, R Stanley, S-T Yau, editors), Int. Press, Somerville, MA (2011) 45-93 MR2906371

[17] C-H Sah, Homology of classical Lie groups made discrete, III, J. Pure Appl. Algebra 56 (1989) 269-312 MR982639 
[18] P Salvatore, Configuration spaces with summable labels, from: "Cohomological methods in homotopy theory", (J Aguadé, C Broto, C Casacuberta, editors), Progr. Math. 196, Birkhäuser, Basel (2001) 375-395 MR1851264

[19] D P Sinha, Manifold-theoretic compactifications of configuration spaces, Selecta Math. 10 (2004) 391-428 MR2099074

[20] D P Sinha, Operads and knot spaces, J. Amer. Math. Soc. 19 (2006) 461-486 MR2188133

[21] D P Sinha, The topology of spaces of knots: cosimplicial models, Amer. J. Math. 131 (2009) 945-980 MR2543919

[22] A A Suslin, On the $K$-theory of local fields, from: "Proceedings of the Luminy conference on algebraic $K$-theory”, (E M Friedlander, M Karoubi, editors), volume 34 (1984) 301-318 MR772065

[23] V Turchin, Hodge-type decomposition in the homology of long knots, J. Topol. 3 (2010) 487-534 MR2684511

[24] MS Weiss, Orthogonal calculus, Trans. Amer. Math. Soc. 347 (1995) 3743-3796 MR1321590

[25] MS Weiss, Embeddings from the point of view of immersion theory, I, Geom. Topol. 3 (1999) 67-101 MR1694812

[26] MS Weiss, Erratum to the article Embeddings from the point of view of immersion theory: Part I, Geom. Topol. 15 (2011) 407-409 MR2776849

Department of Mathematics, Kansas State University

138 Cardwell Hall, Manhattan, KS 66506, USA

turchin@ksu.edu

http://www.math.ksu.edu/ turchin/

Received: 8 October 2012 Revised: 30 December 2012 